\title{
Erratum: Non-Gibbs states on a Bose-Hubbard lattice [Phys. Rev. A 99, 023603 (2019)]
}

Alexander Yu. Cherny, Thomas Engl, and Sergej Flach

Q (Received 4 March 2020; accepted 6 March 2020; published 17 March 2020)

DOI: 10.1103/PhysRevA.101.039901

We wish to correct misprints and errors of the original paper.

(1) Equations (11) and (33) are misprinted and should read

$$
\begin{aligned}
\ln Z(\beta, \alpha, M) & =\ln Z_{0}(\alpha, M)-\beta\langle\hat{H}\rangle_{0}+\beta^{2}\left(\left\langle\hat{H}^{2}\right\rangle_{0}-\langle\hat{H}\rangle_{0}^{2}\right) / 2+\cdots, \\
W_{\mathrm{cl}}(E, N, M) & =\left(\frac{N e}{M}\right)^{M} \exp \left[-\frac{M\left(\varepsilon-U n^{2}\right)^{2}}{2 f_{\mathrm{cl}}(n)}\right] .
\end{aligned}
$$

(2) The two paragraphs around Eq. (42) should read as

"It is straightforward to extend the hopping network between sites. For instance, for binary interactions, a one-band Hamiltonian takes the form

$$
\hat{H}=\sum_{i \neq j} J(i-j) \hat{a}_{i}^{\dagger} \hat{a}_{j}+\frac{1}{2} \sum_{i, j} U(i-j) \hat{a}_{i}^{\dagger} \hat{a}_{j}^{\dagger} \hat{a}_{j} \hat{a}_{i} .
$$

Using the same method as in Sec. II, we arrive at non-Gibbs states above the line $\varepsilon=U_{0} n^{2}$ with $U_{0} \equiv U(0)+\frac{1}{2} \sum_{j \neq 0} U(j)$, which is assumed to be finite and positive."

(3) The commutation relation between the phase $\hat{\varphi}_{j}$ and charge $\hat{q}_{i}$ on p. 6 is misprinted and should be written as $\left[\hat{q}_{i}, e^{i \hat{\varphi}_{j}}\right]=\delta_{i j} e^{i \hat{\varphi}_{j}}$.

These corrections do not affect the results and conclusions of the original paper. 